\title{
Faceting of local droplet-etched nanoholes in AlGaAs
}

\author{
Vedran Vonk, ${ }^{1}$ Taras Slobodskyy, ${ }^{2}$ Thomas F. Keller, ${ }^{1,3}$ Marie-Ingrid Richard, ${ }^{4,5}$ Sara Fernández, ${ }^{4,5}$ Tobias Schulli, ${ }^{5}$ \\ Christian Heyn, ${ }^{2}$ Wolfgang Hansen, ${ }^{2}$ and Andreas Stierle ${ }^{1,3}$ \\ ${ }^{1}$ Deutsches Elektronen-Synchrotron DESY, D-22603 Hamburg, Germany \\ ${ }^{2}$ Center for Hybrid Nanostructures CHyN, Universität Hamburg, D-20355 Hamburg, Germany \\ ${ }^{3}$ Fachbereich Physik, Universität Hamburg, D-20355 Hamburg, Germany \\ ${ }^{4}$ Univ Toulon, Aix Marseille Univ, CNRS, UMR IM2NP 7334, Marseille, France \\ ${ }^{5}$ European Synchrotron Radiation Facility, Grenoble, France
}

(Received 27 June 2018; published 8 October 2018)

\begin{abstract}
Nanoholes, drilled in the (001) surface of AlGaAs by local Al droplet etching, are shown to consist of faceted inner walls. The most prominent facets of the inverted pyramidlike nano-sized etch pits are the $\{111\} \mathrm{A}$ and $\{1 \overline{1} 1\} B$ surfaces, which differ in their atomic surface terminations. In the [110] direction, the $\{111\}$ facets change to $\{112\}$ and/or $\{113\}$, which are both stepped surfaces with (111)A terraces. Etching-temperaturedependent data indicate that this facet transition seems kinetically hindered up to etch temperatures above $660{ }^{\circ} \mathrm{C}$, at which point the walls along [1 $1 \overline{1} 0]$ have already evolved completely towards $\{1 \overline{1} 1\} \mathrm{B}$ facets. The redeposited ring material outside the nanohole develops facets with indices of (115) and higher, thereby forming relatively flat structures. The facets and their indices are unraveled by a combination of atomic force microscopy, scanning electron microscopy, and x-ray diffraction, which is performed on an ensemble as well as on a single hole using nanodiffraction. These results imply that this nanoconfined etching process can be largely understood in a vapor-liquid-solid scheme, which includes the bulk thermodynamics in the Al-Ga-As system, the surface energies of low index facets, and their etch rates and surface terminations.
\end{abstract}

DOI: 10.1103/PhysRevMaterials.2.106001

\section{INTRODUCTION}

Semiconductor nanostructures hold promise, e.g., for their use in quantum information technology [1] and nanoscale optoelectronics. In particular, semiconductor quantum dots (QDs) represent building blocks [2] as qubits for quantum computing [3,4] or as sources for entangled photons [5-7] for quantum communication.

Several techniques are employed for QD fabrication such as lithography [8], field-effect definition [9], chemical synthesis [10], and epitaxial self-assembly. For the epitaxial selfassembly, strain-driven methods [11-14] and droplet epitaxy based techniques [15-20] are used. An alternative fabrication method comprises the local droplet etching (LDE) of semiconductor surfaces [21-25], by which self-assembled nanoholes are formed. Filling of nanoholes in AlGaAs allows for the creation of strain-free GaAs QDs [26,27]. Aside from single QDs, also vertically stacked quantum dot molecules with strong coupling [28] and ultrashort nanopillars [29] for thermoelectric applications are fabricated by filling of LDE nanoholes.

Etching of semiconductors has a long tradition and is an important processing technology. Controlling and understanding of etching in nanoconfinement has gained ever more importance in view of the fabrication of nanotubes from a coreshell nanowires [30] or complicated vertical [31] structure and during the congruent melting of GaAs [32] and subsequent quantum-ring formation [33]. To a large extent, the fabrication and etching of such structures can be understood by considering thermodynamic aspects in the vapor-liquid-solid (VLS) mechanism [34], which includes surface energies, chemical potentials, and the complicated interplay between them [35].

The crystallographic shape of the nanoholes is a key ingredient for the further understanding of the mechanism behind droplet etching and their application as a template for nanostructure creation. The main characterization technique addressing the shape of the LDE nanoholes is ex situ atomic force microscopy (AFM) in air. In addition, cross-sectional transmission electron microscopy (TEM) has been performed [36] on AlAs samples, which are highly reactive towards fast oxidation. In the case of AFM, the tip convolution effect is very often unknown for that particular measurement, which hampers the detailed shape analysis. Sample preparation for TEM is very complicated because one needs to cut a very thin slice from the sample exactly through a hole. Finding an appropriate QD in this procedure is highly time-consuming since the holes have a low density of $10^{7}-10^{8} \mathrm{~cm}^{-2}$. At the same time, once the hole has been prepared in a certain cut direction, information on the other directions is lost. By combining different methods on the same sample, some of these issues can be overcome, because it allows for validation and cross comparison of results.

Here we use a combination of AFM, scanning electron microscopy (SEM), and x-ray diffraction. With AFM the height profile around the hole and the depth of single holes can be determined. From the SEM measurements the lateral structure can be determined with a high resolution. Accurate crystallographic information on the faceting directions can be obtained from x-ray diffraction. In the vicinity of the Bragg peaks, streaks of scattered intensity are observed in the 
directions perpendicular to the facets. The appearance of such scattering signals has already been described by von Laue in 1936 [37] and has been used even more in recent years with the advances in diffraction from nanoscaled objects. For example, ensemble-averaged scattering signals from supported $\mathrm{Rh}$ nanoparticles have uncovered shape changes during $\mathrm{CO}$ oxidation cycles [38] and coherent diffraction imaging (CDI) $[39,40]$ of single nanowires can give detailed information on their shape and strain state [41]. The application of $\mathrm{x}$ rays allows for a nondestructive $3 \mathrm{D}$ view of the nanoholes which can also be used for future in situ investigations with a dedicated ultra-high vacuum (UHV) chamber.

The most important result of our study of an Al-etched AlGaAs film is that the inner walls are formed by facets with relatively low indices, which represent the energetically most stable directions. Temperature-dependent nanohole characterization implies that around $660^{\circ} \mathrm{C}$ the LDE process has evolved towards and possibly reached thermodynamic equilibrium, which is discussed in terms of the surface energies and the ternary Al-Ga-As system.

\section{EXPERIMENT}

The samples were grown in a solid-source molecular beam epitaxy (MBE) system equipped with a valved cracker source for $\mathrm{As}_{4}$ evaporation. Growth is performed on epiready $\mathrm{GaAs}(001)$ wafers. The crystallographic directions on the wafer are according to the manufacturer's specifications and follow the general convention for GaAs [42]. The layer sequence of the investigated sample is sketched in Fig. 1(a). This multilayer structure allows for investigating local droplet etching on more complicated heterostructures, which are needed for real applications. For example, the nanoholes can be used as a template for QD fabrication and a highly doped layer can easily be incorporated for control of the electron occupation in the dot and the electric field at its location. Al droplets are used for etching of nanoholes into the topmost AlGaAs layer, which results in optically inactive AlAs walls around the nanohole opening [26]. For droplet etching, the As flux is reduced by a factor of approximately 100 compared to GaAs growth. This is achieved by closing the valve and shutter of the As cell as well as the main-shutter in front of the sample surface. Afterwards, 1.8 monolayers (ML) of Al are deposited at temperatures in the range of $T=570-680^{\circ} \mathrm{C}$. The deposited Al forms droplets in Volmer-Weber growth mode [43]. During a postgrowth thermal annealing step at unchanged temperature the droplets transform into nanoholes surrounded by a crystalline AlAs wall. Details of the process and discussions of the mechanisms behind droplet etching are given in previous publications [23-25].

The sample surfaces are investigated using AFM in tapping mode under air. We have not noticed any severe morphological degradation for the samples presented here, neither when keeping them in inert nitrogen nor when exposing them to air. This is in contrast to holes drilled in AlAs, which contain more aluminum and are unstable after air exposure. An example of an AFM image from the sample with low-density, spatially well-separated nanoholes in an atomically flat AlGaAs surface is shown in Fig. 1(b).
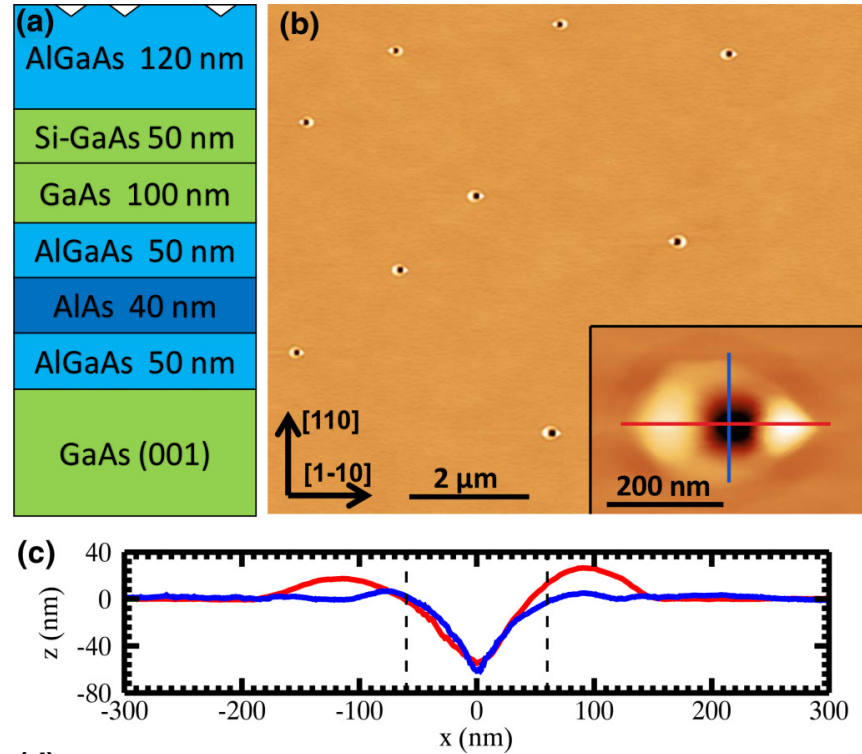

(d)

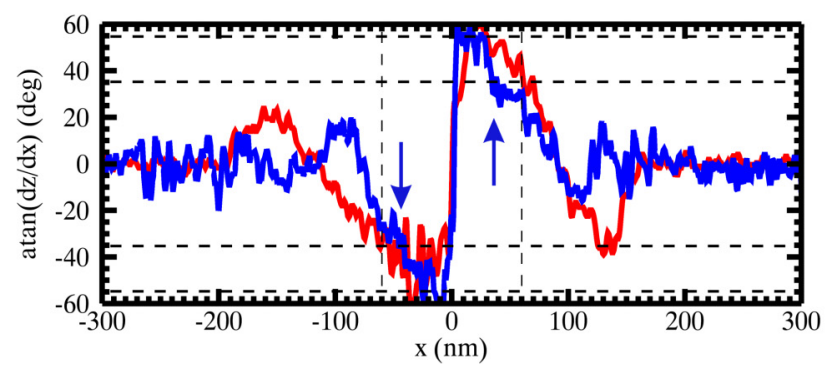

FIG. 1. Overview of the sample etched with $\mathrm{Al}$ at a temperature of $T=625^{\circ} \mathrm{C}$. (a) Cross-sectional schematic of the multilayer structure with $\mathrm{Al}$ droplet etched nanoholes in the topmost layer. The Al content in the AlGaAs layers is 0.35. (b) AFM image of the sample surface with nanoholes of low density $\left(1 \times 10^{7} \mathrm{~cm}^{2}\right)$. The inset shows a magnified hole illustrating the anisotropic shape of the wall around the nanohole. (c) AFM line scans through the hole center along [110] (blue) and [110] (red) directions with equal $x$ and $z$-axis scales. The vertical dotted lines indicate the extension of the nanohole. (d) Derivatives of the AFM line scans shown in (c). The vertical dotted lines indicate the extension of the nanohole and the horizontal dotted lines indicate the angular values of $\{111\}$ facets $\left(55^{\circ}\right)$ and $\{112\}\left(35^{\circ}\right)$. The arrows indicate the positions where the slope abruptly changes along the [110] direction. This plot further shows that inside the hole, where material was etched, the steepest walls occur. Most of the surface of deposited material outside the nanohole has much flatter orientations.

Scanning electron microscopy was performed at the Desy Nanolaboratory [44] using a field emission instrument. The results presented here were obtained by recording secondary electrons with a through-lens detector.

$\mathrm{x}$-Ray diffraction experiments have been performed at beamlines ID03 and ID01 of the European Synchrotron Radiation Facility. At ID03, the $30 \times 100 \mu \mathrm{m}^{2}$ x-ray beam of energy $E=9.5 \mathrm{keV}$ impinged on the sample at a grazing angle of $0.2^{\circ}$, thereby creating an approximately $1-\mathrm{mm}^{2}$ footprint on the sample surface. The ID03 measurements therefore represent an ensemble average over approximately $10^{5}$ nanoholes. At ID01, the nanofocused beam of $300 \times 300 \mathrm{~nm}^{2}$ 


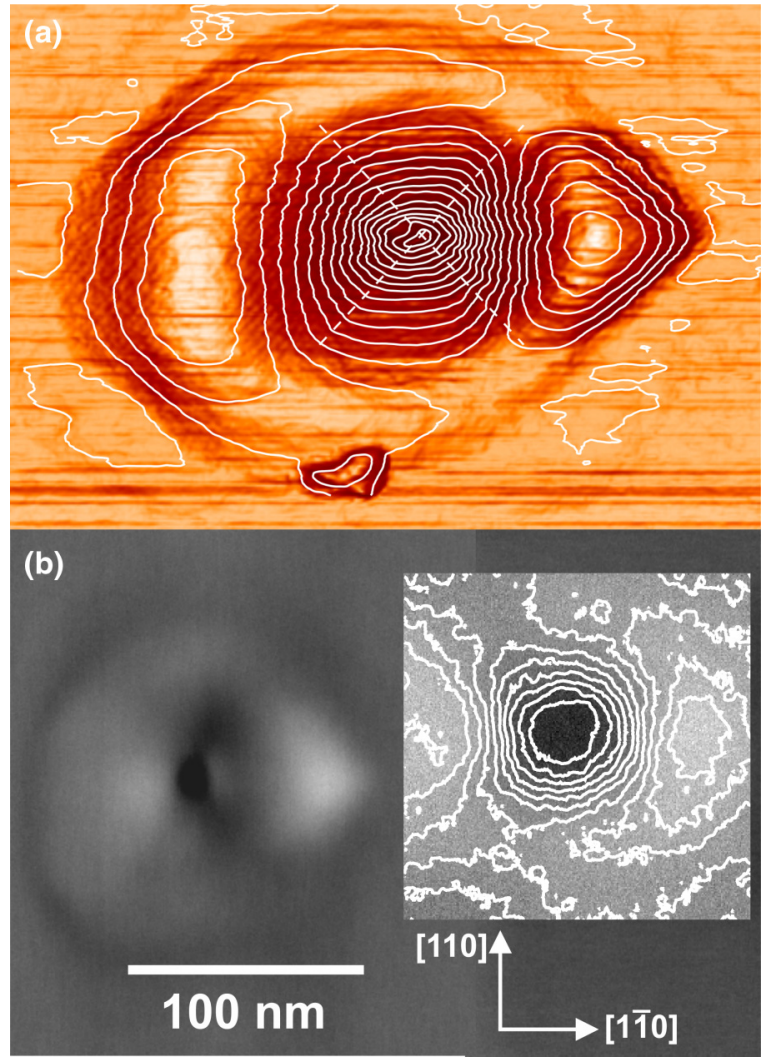

FIG. 2. Microscopy results. (a) AFM of a single nanohole as in Fig. 1. The solid contour lines highlight the presence of inner facets. The dashed lines indicate the edges between the different facets. Due to the fact that the hole is not exactly square, these edges are also not at exactly $90^{\circ}$ to each other. This is explained by the inner facet wall changing from (111) to (112) along the [110] direction. (b) High-resolution SEM of a single nanohole and ring. The inset shows contour lines, which highlights the nearly square shape and the presence of inner facets.

size and energy $E=8.0 \mathrm{keV}$ allowed us to illuminate a single nanohole at an angle of incidence of $50^{\circ}$.

\section{RESULTS}

\section{A. Microscopy}

AFM measurements in Fig. 1(b) show that the density of the LDE holes is approximately 1 hole per $10 \mu \mathrm{m}^{2}$. The inset in Fig. 1(b) shows a magnification of a hole with a nearly square hole opening. This might already indicate the formation of crystallographic facets inside the hole, which is further highlighted by overlaying contour lines [Fig. 2(a)]. The ringlike structure surrounding the hole is also clearly imaged with high-resolution SEM, see Fig. 2(b), having an estimated resolution of 2-3 nm. Both microscopy images show that the [110] and [110] directions display different shapes. The line profiles across the AFM images, see Fig. 1(c), show that the inner walls have different shapes with respect to the [110] and [110] directions. By analyzing the derivative of the line profiles, as is seen in Fig. 1(d), the steepness of the inner walls falls in the range of low index $\{111\}$ - and $\{112\}$-type facets. Particularly notable is that the slope of the inner wall along the [110] remains higher and closer to $55^{\circ}$ than the slope along [110], which suddenly drops to a value close to $35^{\circ}$ after about $30 \mathrm{~nm}$. The lateral extension of this constant slope is large enough to be resolved by AFM, which has an estimated resolution of about $10 \mathrm{~nm}$. This seems to indicate that the inner wall along the [1110] direction on one side of the nanohole consists of a (111) facet, whereas along the [110] direction it changes from a (111) facet close to the bottom to a (112) facet close to the top. On the other side (negative $x$ values), a similar, but less clear, trend exists, whereby the slope along the [110] direction shows a sudden drop at about $-40 \mathrm{~nm}$. The relatively noisy derivative patterns alone make it difficult to clearly distinguish the presence of low-index facets. The $(h k l)$ assignment is done in tandem with the x-ray results presented hereafter and by assuming that the average direction of the inner walls consists of such low index facets, rather than high index (miscut) orientations, which would energetically be less favorable. Whereas the slopes for $\{111\}$-type surfaces seem to fit rather well, the (1112) facet slope is actually somewhat smaller than $35^{\circ}$ but still larger than $25^{\circ}$, which corresponds to a (113) facet. Either the used method is not accurate enough or it is indeed a high-index facet, like, e.g., (225). This particular issue will be discussed in detail below. Nevertheless, the inner wall in the [110] direction does change its slope, as discussed in relation to the growth temperature in Sec. III C.

Both the AFM and SEM results indicate that the hole opening has a nearly square shape, larger by a few $\mathrm{nm}$ in the [110] direction. This is consistent with the presence of the $\{112\}$ or $\{113\}$ facets, which have a smaller slope than $\{111\}$. The nearly square shape also implies that the inner faceted walls meet at well-defined edges, as highlighted in Fig. 2(a), just as this is known for faceted crystals and nanoparticles.

\section{B. $x$-Ray diffraction}

Figure 3 shows reciprocal space maps (RSMs) around the GaAs 111 Bragg reflection of the Al-etched sample. Two mutually perpendicular planes parallel to the [110] and [1 $\overline{1} 0]$ directions are mapped out. The relatively large beam $(30 \times$ $100 \mu \mathrm{m}^{2}$ ) illuminated about $10^{5}$ holes. These maps therefore represent the ensemble average. The inhomogeneous, butterfly-like intensity distribution around the central crystal truncation rod (CTR) arises due to scattering from facets. By common convention, the GaAs(001) surface is perpendicular to the $L$ direction. The relatively strong $(1,1)$ CTR from the $\mathrm{GaAs}(001)$ surface is therefore seen as a streak along $L$ (the difference in width in the two maps arises from scattering resolution effects). Any surface (facet) that is not parallel to the main $\operatorname{GaAs}(001)$ will create its own CTR-like streak along a direction perpendicular to this facet. The intensity of the CTR scales linearly with the surface area from which it arises; this explains that the small facets around and inside the nanoholes will lead to much weaker streaks compared to the CTR of the main $\operatorname{GaAs}(001)$ surface. The width of a facet-CTR perpendicular to its streak direction scales inversely with the lateral facet size in that direction: Small facets will lead to broad CTRs in reciprocal space. This general diffraction effect may also obscure the unequivocal determination of different facets due to overlap of their CTRs in reciprocal space. For flatter facets, i.e., streaks with ever-higher $L$ indices, it is getting 


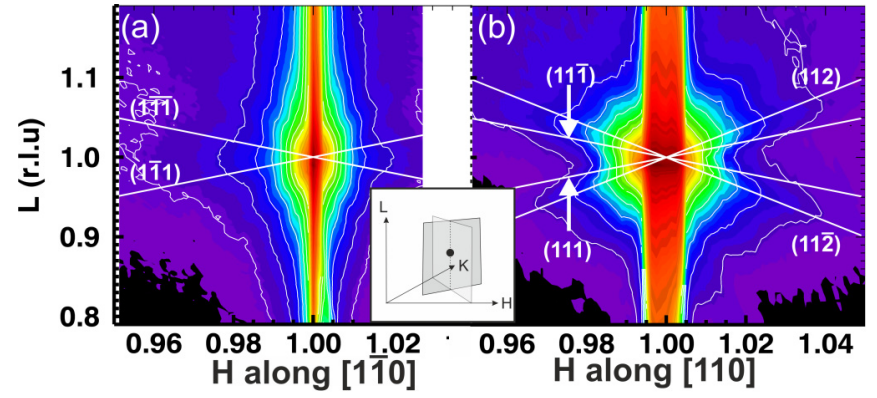

(c)

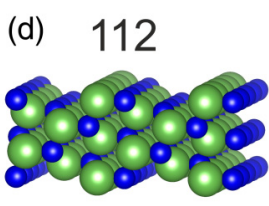

(e)

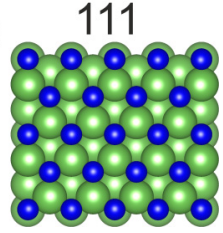

FIG. 3. Ensemble reciprocal space maps around the $111 \mathrm{GaAs}$ Bragg peak. (a) Map in the $(H+K=2, L)$ plane, which is perpedicular to the $(H, H, L)$ plane. The inset shows schematically the planes mapped out in reciprocal space around the Bragg peak (black dot). The weak, nonuniform butterfly-like intensity distribution around the central Bragg peak arises due to scattering from facets. The directions (white lines) and indices of the steepest observed facets, as discussed in the text, are indicated. (b) Map in the $(H, H, L)$ plane. The directions (white lines) and indices of the steepest facets are indicated. (c) Atomic-scale surface model showing Ga (blue) and As (green) of the (11̄1)B surface. (d) The (112) surface is stepped with close-packed, Ga-terminated (111)A terraces and As-terminated (001) steps. (e) The (111)A Ga-terminated surface is shown in the top view.

ever more difficult to precisely distinguish their directions. For example, it is difficult to unequivocally assign the steepest streak in the [11L] map (Fig. 3) to (115) or (116) or even a combination of the two. From the AFM results, presented in Sec. III A, it is seen that the inner walls of the nanoholes are the steepest, which could be formed by (111) and (112) facets. The RSM in the $(H+K=2, L)$ plane clearly shows an intensity streak along the $(1, \overline{1}, 0)$ direction, which arises

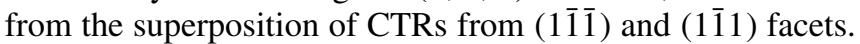
Based on the microscopy results, the presence of (110) facets is ruled out. These streaks are relatively well separated from other streaks, which have a smaller angle to the $(1,1)$ CTR direction, thereby showing that they result from the steepest facets. The situation is different for the RSM in the $(H, H, L)$ plane showing rather well-developed streaks along the $(1,1,2)$ and $(1, \overline{1}, 2)$ directions, which somewhat obscure the $(1,1,1)$ and $(1, \overline{1}, 1)$ streaks. This scattering pattern is completely in line with the AFM direct space observation (Fig. 1), which shows that the nanoholes consist of (111) facets at the bottom and (112) at the top. This leads to a superposition of streaks, whereby each contribution is weighed by the surface area of the corresponding facet. Assuming an inverted pyramidal shape which is cut at half height, the ratio of the outer areas between the bottom and top is $1: 3$. This means that the (112) facet area close to the top of the nanohole is approximately 3 times larger than the (111) facet area at the bottom, and it is expected that the corresponding scattering is also 3 times stronger, thereby showing up more prominently in the RSM. The ring material outside the nanoholes contains much flatter
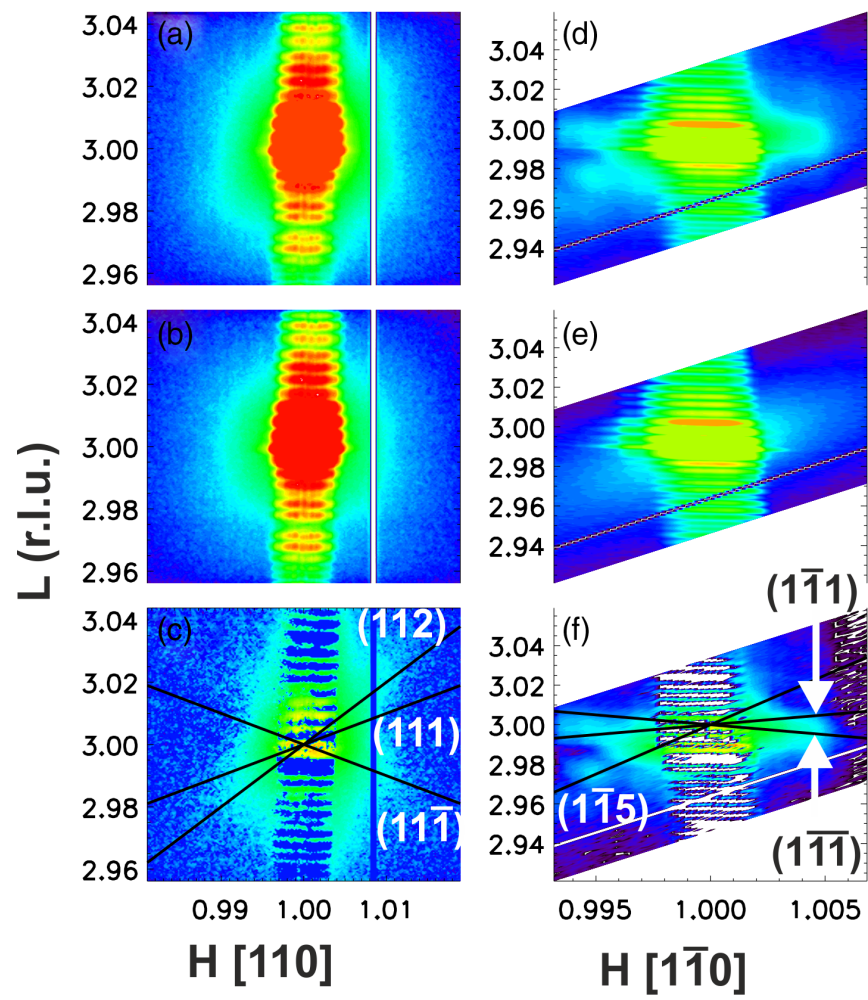

FIG. 4. Single hole reciprocal space maps around the $113 \mathrm{GaAs}$ Bragg peak using a 300-nm x-ray beam. Shown are the $(H, H, L)$ [(a)-(c)] and $(H+K=2, L)$ planes [(d)-(f)]. Data were taken with the X-ray beam aligned on the nanohole [(a) and (d)] and in between the holes [(b) and (e)]. The difference maps [(c) and (f)] highlight the weak nonuniform intensity distribution around the central Bragg peak which arises due to scattering from facets, the directions (black lines) and indices of which are also indicated. The other lines [vertical in (a)-(c) and diagonal in (d)-(f)] originate from the x-ray insensitive junctions between neighboring chips on the detector.

surfaces, which leads to streaks that are much more parallel to the GaAs $(1,1)$ CTR in reciprocal space. This observation is important in order to distinguish which facets were formed during the etching process and which ones by redeposition of material. It can also be seen that the two directions, in which the maps were recorded, show different intensity distributions, which points to different facet directions and/or different facet area sizes. The holes and rings are anisotropic with respect to the [110] and [110] directions, not only in shape, as seen in the microscopy images, but also in type of facets that have formed. The ensemble-averaged maps of many holes show relatively well-defined facet scattering, which indicates that there is not a very large spread in the crystallographic shape between different holes.

Figure 4 shows RSM results around the 113 Bragg peak for a single hole when using a nanobeam. This Bragg peak was chosen because of the favorable scattering geometry, which for this reflection was such that the incident beam made an angle of $50^{\circ}$ and diffracted beam approximately $4^{\circ}$ with the surface. Hereby the x-ray beam's footprint was small, in contrast to grazing incidence geometries. Measurements are shown whereby the x-ray beam illuminated a single hole and when it was aligned in between the holes on the bare substrate. The 
resulting diffraction patterns clearly show differences, which we ascribe to scattering from facets. Again, the steepest facets, which have intensity streaks the furthest away from the [001] direction, are found in the $(H+K=2, L)$ plane, agreeing very well with the ensemble-averaged measurements. The RSM in the $(H+K=2, L)$ plane from a single hole shows a more asymmetric intensity distribution than the map obtained from many holes. The similarity between the $(H, H, L)$ maps from the single hole and the ensemble is much greater and the intensity distributions are much more symmetric than in the $(H+K=2, L)$ planes. A first explanation comes from the fact that the nanoholes appear in two different orientations on the surface, as can be seen in Fig. 1(b). The triangular island on the ring is sometimes observed on the right side and sometimes on the left of the hole along the [110] direction. This bimodal orientation distribution will give rise to an apparent pmm symmetry in the ensemble-averaged diffraction pattern, which is obviously seen in Figs. 3(a) and 3(b) but which is not present in the case of diffraction from a single hole. A single hole and ring seem to have symmetry pm, with the mirror plane along the [11 0 ] direction. This symmetry argument needs to be combined with the inherent intensity distribution along the streak, which can be asymmetric with respect to the Bragg peak due to the details of the atomic structure of that particular facet surface. For example, relaxation perpendicular to the facet surface will lead to scattering interference effects which make the shape of the facet-CTR asymmetric. Such details might get lost in an ensemble average but not in the single hole diffraction measurement. The RSM in the $(H+K=2, L)$ plane [Fig. 4(f)] shows one particular feature in the $(1, \overline{1}, 5)$ direction, which is not related to the inner walls but to the ring material. Since the holes were drilled with $\mathrm{Al}$, strain effects are not expected to play an important role and this small peak is not related to Bragg scattering from the ring material. Most likely, it is a fringe which appears from finite-size diffraction in that direction. A complete explanation cannot be given at the moment. Similarly, the spacing of the fringes seen in the maps along the $L$-direction correspond to a thickness of approximately $85 \mathrm{~nm}$. This does not correspond to a particular layer from the stack of deposited materials (see Fig. 1). The same fringe spacing is seen when the beam is illuminating a nanohole and when it is in between them. For the moment it remains unclear where this interference pattern originates from.

Another reason for the fact that not all the facets observed in the ensemble-averaged maps appear in the ones from the single hole is because of a lack of scattered intensity. In the case of the ensemble average, the scattered intensity is proportional to the number of illuminated holes and leads to a huge amplification in the case that all the holes have identical shape. A next difference between the maps can arise due to the fact that since the nanobeam footprint was nearly the same size as the nanohole and surrounding ring, slight misalignment can lead to parts being missed, whereby especially the flatter facets of the ring could be affected.

\section{Temperature evolution}

The detailed crystallographic shape analysis presented in the previous sections was done on a single sample grown at
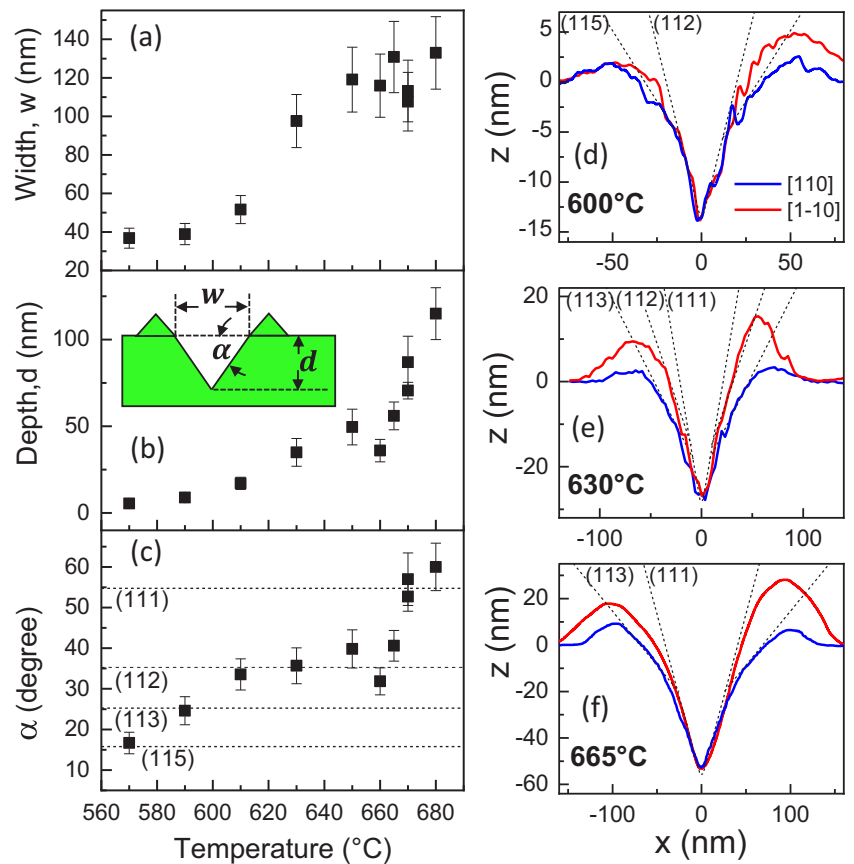

FIG. 5. AFM results of the average nanohole shape at varied process temperature $T$. Shown are the average values of different shape parameters, which are obtained from multiple measurements on different holes. The error bars represent the scatter in the obtained values. (a) Width of the nanohole opening averaged over the [110] and [1피 directions. (b) Nanohole depth $d$ giving the distance between the hole bottom and the planar surface. The inset shows a cross-sectional sketch of a hole with the characteristic sizes. (c) Average hole side-facet angle $\alpha$ calculated from the the hole width and depth. The values for various $(11 n)$ facets are also indicated (dotted lines). (d) AFM profiles along [110] and [110] directions from a nanohole fabricated at $T=600^{\circ} \mathrm{C}$, (e) a nanohole fabricated at $T=630^{\circ} \mathrm{C}$, and (f) a nanohole fabricated at $T=665^{\circ} \mathrm{C}$. The (111)-, (112)-, and (113)-type facets are indicated for comparison (dotted lines).

$625^{\circ} \mathrm{C}$. Here we address the link with the thermodynamic equilibrium and kinetics aspects of GaAs etching in general and LDE in particular. To this end, the AFM shape analysis results of a series of Al-drilled samples fabricated at diffferent temperatures is presented. This comparison also allows us to verify the reproducibility of sample preparation and hole-to-hole differences. Since for this series of samples only AFM data are available, a similar detailed analysis as presented in the previous sections is not possible. Therefore, we revert to a more general description, as given by assuming a conelike shape [24]. Such a description does not explicitly include low-index facets but merely the average steepness of the hole walls. A sketch of the quantities used for such hole characterization is shown in the inset of Fig. 5(b). In detail, the average width $w$ along [110] and [11 10 ] of the hole opening and the hole depth $d$, giving the distance between the hole bottom and the planar surface, are determined from AFM line scans and together define the average wall angle $\alpha$. In this way, detailed information on the existence of possible low-index facets is averaged out. Figures 5(a) and 5(b) show results from a sample series where the temperature $T$ during 
the LDE process was varied [24]. Obviously, a higher $T$ yields deeper holes with a larger diameter. The average side facet angle in cone approximation is plotted in Fig. 5(c) together with values of several crystallographic facets. The values presented in Fig. 5 are obtained by averaging over several holes and the indicated errors represent the scatter. The hole depth $d \simeq 60 \mathrm{~nm}$ and width $w \simeq 120 \mathrm{~nm}$ of the $625^{\circ} \mathrm{C}$ sample that is most extensively studied here, represent the upper end of the values presented in Fig. 5 near that growth temperature. Nevertheless, these structural parameters still lie within the relatively large error bars and follow the general trend with temperature. The data show how the $\{111\}$ and $\{1 \overline{1} 1\}$ facets become more pronounced with increasing etching temperature. From the value of $\alpha$ shown in Fig. 5(c), it follows that with increasing temperature the average steepness of the inner walls rises from, on average, approximately a $\{113\}$ to a $\{111\}$-type facet. Nevertheless, in the [110] direction, there still is a transition to the flatter, approximately (113), facet, as seen in Fig. 5(f). With increasing temperature, there are two transitions to be seen. In the range $T=620-640{ }^{\circ} \mathrm{C}$, the width increases from approximately 50 to $120 \mathrm{~nm}$, while the hole depth remains nearly constant. This is for a large part attributed to the lateral droplet size, which increases due to Ostwald ripening around this temperature [24]. Around $T \simeq 660^{\circ} \mathrm{C}$, suddenly also the hole depth increases from approximately 50 to $100 \mathrm{~nm}$. This seems to indicate that around $660^{\circ} \mathrm{C}$ some kinetic etching barrier has been overcome, which also correlates with the observation of ever more of the most stable $\{111\}$ and $\{1 \overline{1} 1\}$ facets.

\section{DISCUSSION}

The clear observation of mostly $\{111\}$-type facets after the LDE process, as presented here, implies that the shape of the formed holes is not far off its equilibrium shape. In a thermodynamics picture, this can be understood by the fact that after etching the resulting shape is determined by the most stable facets, which have the lowest etch rates. Although kinetics play a crucial role, as discussed hereafter, apparently, for the LDE process, there is a narrow temperature window in which the kinetics are fast enough to evolve towards this equilibrium shape. Etching of semiconductors with a zincblende structure is an important technology and the crystal habit or etch pit shape can be described by the so-called Jaccodine-Wulff construction [45], which graphically depicts the etch rates as a function of polar crystal orientations. To a very good approximation, it has been shown that the etch pit shape can be understood already by considering only the etch rates of a few key orientations [46]. In the case presented here, this means that only the slowest etching $\{111\}$ directions need to be considered. For the zinc-blende structure, it is known that the etch rates of the so-called (111)A and (111)B facets are different, because they terminate with a different atomic layer along this polar stacking direction [47] and also have a small difference in surface energy [48]. Combining all these considerations for the LDE process of AlGaAs, the true equilibrium nano-etch pit shape will, to a good approximation, consist of two $\{111\}$ A by two $\{1 \overline{1} 1\} \mathrm{B}$ facets forming an inverted pyramid with a square base, which is the hole opening. Due to the symmetry of the crystal lattice, two

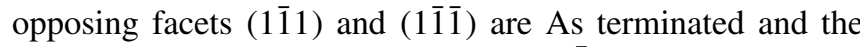
other two opposing facets (111) and (11) $)$ are Ga terminated [see also Figs. 3(c)-3(e)]. The different terminations of the facets in the [110] and [1 10$]$ directions introduce a slight anisotropy and this can partly explain differences seen in the nanohole's shape in the two directions. Etching $\mathrm{Si}(001)$, which does not contain a polar stacking sequence, through a mask is known to produce symmetric inverted pyramidal etch pits consisting of $\{111\}$ walls [49]. During the LDE process, material is removed. In a simplified picture, one can consider only the formation of $(11 n)$ and $(1 \overline{1} n)$ facets. For large values of $n$ the etch rates are fast. The final facet that is formed, with the lowest etch rate, will be for $n=1$. The difference between $(11 n)$ and $(1 \overline{1} n)$ facets is the surface termination. For example, the stepped surface (112) consists of (111)A terraces while (11) consist of (111)B terraces. In general, it is expected that the As-terminated surfaces will etch faster, which means that for each $n$ the $(1 \overline{1} n)$ etch rate is expected to be faster than for $(11 n)$. This may explain the observation of the (112) facet, which most likely has only a somewhat faster etch rate than (111) and is therefore still present once the LDE process has finished. In the same period of time, the faster etching (112) has already disappeared, leaving only the (11) facet in this direction. Unfortunately, GaAs etch rates for different facets are reported in the literature mostly for alkaline solutions, which makes it difficult to compare with the case of using aluminium to remove material.

The accuracy of the methods presented here cannot rule out the presence of other facets, which would have orientations in between the ones discussed so far. High-index planes have been observed on GaAs and can even be as stable as low-index ones [50-52]. The driving force for the existence of such surfaces is the minimization of the number of dangling bonds and strain. Whereas this reasoning is rather straightforward for surfaces in vacuum, it becomes more complicated at solid-liquid interfaces, which is the case during local droplet etching. It is conceivable that the presence of a liquid metal may immediately saturate any dangling bonds, which changes the energetics picture drastically. Indeed, already deposition of the order of $1 \mathrm{ML}$ of $\mathrm{Ga}$ or As on AlGaAs changes the surface reconstructions [53], for which dangling bonds and strain play a big role. There are also reports for the $\operatorname{Si}(111)$ surface, buried under liquid indium [54] or lead [55], which do not observe a reconstructed surface at the interface. These examples are important when discussing the stability of surfaces in contact with liquids and illustrate why in first-order approximation we do not assume high-index facets to have formed.

The actual sequence of processes which lead to the final nanohole shape and composition are more complicated to predict. The thermodynamic driving force can in first instance be rationalized by the ternary Al-As-Ga system [56]. The initial situation, namely that of a pure Al liquid (the droplet) in contact with an AlGaAs solid (substrate), does not represent equilibrium and will lead to dissolution of the solid. The system strives to reach equilibrium, which consists of a solid-liquid interface, where both sides have a particular composition as can be taken from the corresponding tie-lines in the ternary phase diagram. It is important to realize that these compositions can both be different from the initial ones, i.e., both the liquid as well as the solid compositions can 
change. For the liquid this is done by dissolving the solid. The composition of the solid substrate cannot easily change, but redeposition of a film on top of the substrate can lead to the solid-liquid interface required by thermodynamics. Such a process of simultaneous dissolution and precipitation has been previously described for the case of Ge liquid phase epitaxy on Si from an In solution [57]. The final state in such a ternary system where the solid is stable continuously for a whole range of compositions will in general be such that the substrate is overgrown by a thin layer of mixed composition in equilibrium with a liquid of mixed composition. Still, kinetics will determine how and whether this particular equilibrium can be reached and a film deposited by this process will in general have a composition gradient since the system is changing all the time. Here, etching into AlGaAs can lead to the formation of relatively well-defined facets, but at the same time there will be a driving force for redeposition of a thin film onto these facets. How this process exactly evolves is difficult to predict, but apparently it starts only after the etching has evolved to a point where the liquid reaches a particular (critical) composition. Eventually, the composition of the liquid changes even so much that it solidifies, thereby forming the ring material. Most likely this process is largely influenced by the loss of As in the process. This sequence of events could lead to the inner facets being covered by a thin (several $\mathrm{nm}$ ) layer of AlGaAs, which has a different composition from the underlying substrate but also from the material that crystallized from the liquid and most likely forms the ring surrounding the nanohole. These considerations are important when evaluating, for example, the photoluminescence properties of the quantum structures. The exact endpoint of the LDE process will strongly depend on the rates of each of the processes involved, namely etching, As out-diffusion, and redeposition. The surface anisotropy also plays a role in the kinetics. For example, it has been shown that GaAs islands grown on $\mathrm{GaAs}(001)$ are elongated and about 7 times larger in the [110] than in the [110] direction [58]. Such anisotropic mass transport could explain the fact that the ring is much thicker along the $[1 \overline{1} 0]$ direction.

\section{CONCLUSION}

Nanoholes formed in AlGaAs by LDE have been characterized using AFM, SEM, as well as ensemble and single hole x-ray diffraction. The combination of real and reciprocal space techniques allows for circumventing particular data in- terpretation problems that would arise when only one of them would have been used. The picture of the nanohole's shape formation implied from our results is of that of etch-pit wall faceting. The sample that is extensively studied here is grown at $T=625^{\circ} \mathrm{C}$ using $\mathrm{Al}$ droplets and shows $\{111\} \mathrm{A},\{1 \overline{1} 1\} \mathrm{B}$, and $\{112\}$ facets. The redeposited ring material consists of much flatter surfaces, i.e., facets with higher indices, which are clearly seen in the $\mathrm{x}$-ray diffraction and AFM data. A detailed comparison and analysis of AFM data on a series of Al-drilled samples grown at different temperatures, gives the following picture. At temperatures above $665^{\circ} \mathrm{C}$, an inverted approximately pyramidlike etchpit forms, which on average has $\{111\}$-type walls of maximum steepness; this shape is expected in the case of equilibrium. Still, inside the nanohole in the [110] direction, the $\{111\}$ facet changes into $\{112\}$ and/or $\{113\}$. This implies that a kinetic barrier in the [110] direction is still present, whereas it already has been overcome for the formed facets in the faster etching and less stable [1 10$]$ direction. The strictly Ga or As terminations of the facets in both perpendicular directions probably play an important role. The stepped $\{112\}$ and $\{113\}$ surfaces consist of $\{111\}$ A terraces and are therefore considered to be almost equally stable towards Al etching as a planar $\{111\} \mathrm{A}$ surface. Below an etching temperature of $T \simeq 665^{\circ} \mathrm{C}$, the average steepness of the walls diminishes, indicating that flatter facets become more pronounced. Below approximately $620^{\circ} \mathrm{C},\{111\}$-type facets seem not to form at all. These results imply that to a large extent the LDE process can be described by considering thermodynamics and etch rates in different crystallographic directions taking into account the specific atomic terminations.

Moreover, the results presented here are very important if the holes will be used as templates for quantum dot growth. They will help understanding and controlling the symmetry of the holes, which is essential, for instance, in applications in which the dots are sources of entangled photons [7].

\section{ACKNOWLEDGMENTS}

The authors thank the ESRF for providing the beam time and S. Labat for help during the experiment at beamline ID01. W. J. P. van Enckevort, H. Meekes, and G. Bauhuis are acknowledged for fruitful discussions. This project was partially supported by the EU-H2020 research and innovation program under Grant No. 654360, Nanoscience Foundries and Fine Analysis (NFFA), and the Deutsche Forschungsgemeinschaft via HA 2042/8-1.
[1] D. DiVincenzo, Science 270, 255 (1995).

[2] P. Michler, Single Semiconductor Quantum Dots, NanoScience and Technology Series (Springer, Berlin, 2009).

[3] B. Schumacher, Phys. Rev. A 51, 2738 (1995).

[4] S. Michaelis de Vasconcellos, S. Gordon, M. Bichler, T. Meier, and A. Zrenner, Nat. Phot. 4, 545 (2010).

[5] P. Michler, A. Kiraz, C. Becher, W. V. Schoenfeld, P. M. Petroff, L. Zhang, E. Hu, and A. Imamoğlu, Science 290, 2282 (2000).

[6] S. Buckley, K. Rivoire, and J. Vučković, Rep. Prog. Phys. 75, 126503 (2012).
[7] O. Benson, C. Santori, M. Pelton, and Y. Yamamoto, Phys. Rev. Lett. 84, 2513 (2000).

[8] M. C. Rogge, C. Fühner, U. F. Keyser, M. Bichler, G. Abstreiter, W. Wegscheider, and R. J. Haug, Physica E 21, 483 (2004).

[9] L. P. Kouwenhoven, A. T. Johnson, N. C. van der Vaart, C. J. P. M. Harmans, and C. T. Foxon, Phys. Rev. Lett. 67, 1626 (1991).

[10] A. Rogach, S. Kershaw, M. Burt, M. Harrison, A. Kornowski, A. Eychmuller, and H. Weller, Adv. Mater. 11, 552 (1999).

[11] J. M. Moison, F. Houzay, F. Barthe, L. Lepronce, E. Andre, and O. O. Vatel, Appl. Phys. Lett. 64, 196 (1994). 
[12] A. Madhukar, Q. Xie, P. Chen, and A. Konkar, Appl. Phys. Lett. 64, 2727 (1994).

[13] D. Leonard, M. Krishnamurthy, S. Fafard, J. L. Merz, and P. M. Petroff, J. Vac. Sci. Technol. B 12, 1063 (1994).

[14] Y. W. Mo, D. E. Savage, B. S. Schwartzentruber, and M. G. Lagally, Phys. Rev. Lett. 65, 1020 (1990).

[15] T. Chikyow and N. Koguchi, Jpn. J. Appl. Phys. 29, L2093 (1990).

[16] T. Mano, K. Watanabe, S. Tsukamoto, N. Koguchi, H. Fujioka, M. Oshima, C. D. Lee, J. Y. Leem, H. J. Lee, and S. K. Noh, Appl. Phys. Lett. 76, 3543 (2000).

[17] J. S. Kim and N. Koguchi, Appl. Phys. Lett. 85, 5893 (2004).

[18] C. Heyn, A. Stemmann, A. Schramm, H. Welsch, W. Hansen, and A. Nemcsics, Phys. Rev. B 76, 075317 (2007).

[19] M. Abbarchi, C. A. Mastrandrea, T. Kuroda, T. Mano, K. Sakoda, N. Koguchi, S. Sanguinetti, A. Vinattieri, and M. Gurioli, Phys. Rev. B 78, 125321 (2008).

[20] E. Stock, T. Warming, I. Ostapenko, S. Rodt, A. Schliwa, J. A. Töfflinger, A. Lochmann, A. Toropov, S. A. Moshchenko, D. V. Dmitriev, V. A. Haisler, and D. Bimberg, Appl. Phys. Lett. 96, 093112 (2010).

[21] Z. M. Wang, B. L. Liang, K. A. Sablon, and G. J. G. J. Salamo, Appl. Phys. Lett. 90, 113120 (2007).

[22] P. Alonso-González, D. Fuster, L. González, J. Martín-Sánchez, and Y. González, Appl. Phys. Lett. 93, 183106 (2008).

[23] C. Heyn, A. Stemmann, and W. Hansen, Appl. Phys. Lett. 95, 173110 (2009).

[24] C. Heyn, S. Schnüll, and W. Hansen, J. Appl. Phys. 115, 024309 (2014).

[25] C. Heyn, T. Bartsch, S. Sanguinetti, D. Jesson, and W. Hansen, Nanoscale Res. Lett. 10, 67 (2015).

[26] C. Heyn, A. Stemmann, T. Köppen, C. Strelow, T. Kipp, S. Mendach, and W. Hansen, Appl. Phys. Lett. 94, 183113 (2009).

[27] A. Küster, C. Heyn, A. Ungeheuer, G. Juska, S. Tommaso Moroni, E. Pelucchi, and W. Hansen, Nanoscale Res. Lett. 11, 282 (2016).

[28] C. Heyn, A. Küster, A. Ungeheuer, A. Gräfenstein, and W. Hansen, Phys. Rev. B 96, 085408 (2017).

[29] T. Bartsch, M. Schmidt, C. Heyn, and W. Hansen, Phys. Rev. Lett. 108, 075901 (2012).

[30] R. E. Algra, M. Hocevar, M. A. Verheijen, I. Zardo, G. G. W. Immink, W. J. P. van Enckevort, G. Abstreiter, L. P. Kouwenhoven, E. Vlieg, and E. P. A. M. Bakkers, Nano Lett. 11, 1690 (2011).

[31] H. Y. Hui and M. A. Filler, Nano Lett. 15, 6939 (2015).

[32] J. Tersoff, D. E. Jesson, and W. X. Tang, Science 324, 236 (2009).
[33] Z. Y. Zhou, C. X. Zheng, W. X. Tang, J. Tersoff, and D. E. Jesson, Phys. Rev. Lett. 111, 036102 (2013).

[34] R. S. Wagner and W. C. Ellis, Appl. Phys. Lett. 4, 89 (1964).

[35] R. E. Algra, M. A. Verheijen, L.-F. Feiner, G. G. W. Immink, W. J. P. van Enckevort, E. Vlieg, and E. P. A. M. Bakkers, Nano Lett. 11, 1259 (2011).

[36] A. Nemcsics, C. Heyn, L. Toth, L. Dobos, A. Stemmann, and W. Hansen, J. Crystal Growth 335, 58 (2011).

[37] M. Von Laue, Ann. der Phys. 418, 55 (1936).

[38] P. Nolte, A. Stierle, N. Jin-Phillipp, N. Kasper, T. Schulli, and H. Dosch, Science 321, 1654 (2008).

[39] M. A. Pfeifer, G. J. Williams, I. A. Vartanyants, R. Harder, and I. K. Robinson, Nature 442, 63 (2006).

[40] R. Harder and I. K. Robinson, J. Miner. 65, 1202 (2013).

[41] S. T. Haag, M.-I. Richard, U. Welzel, V. Favre-Nicolin, O. Balmes, G. Richter, E. J. Mittemeijer, and O. Thomas, Nano Lett. 13, 1883 (2013).

[42] A. Y. Cho, J. Appl. Phys. 47, 2841 (1960).

[43] M. Volmer and A. Weber, Z. Phys. Chem. 119, 277 (1926).

[44] A. Stierle, T. F. Keller, H. Noei, V. Vonk, and R. Röhlsberger, J. Large Scale Res. Facil. 2, A76 (2016).

[45] R. J. Jaccodine, J. Appl. Phys. 33, 2643 (1962).

[46] Y. Xing, M. A. Gosalvez, H. Zhang, Y. Li, and X. Qiu, J. Microelectromech. Syst. 26, 1063 (2017).

[47] H. C. Gatos and M. C. Lavine, J. Electrochem. Soc. 107, 427 (1960).

[48] N. Moll, A. Kley, E. Pehlke, and M. Scheffler, Phys. Rev. B 54, 8844 (1996).

[49] K. E. Bean, IEEE Trans. Electron. Dev. 25, 1185 (1978).

[50] M. Lopez, T. Ishikawa, and Y. Nomura, Electron. Lett. 29, 2225 (1993).

[51] L. Geelhaar, J. Màrquez, P. Kratzer, and K. Jacobi, Phys. Rev. Lett. 86, 3815 (2001).

[52] R. Mendez-Camacho, V. H. Mendez-Garcia, M. Lopez-Lopez, and E. Cruz-Hernandez, AIP Adv. 6, 065023 (2016).

[53] A. Ohtake, Surf. Sci. Rep. 63, 295 (2008).

[54] V. Vonk, M. Cremers, A. E. F. de Jong, S. Pintea, and E. Vlieg, Surf. Sci. 621, 69 (2014).

[55] C. A. Lucas and D. Loretto, Surf. Sci. 344, L1219 (1995).

[56] C. Li, L. Li, Z. Du, L. Lu, and W. Zhang, J. Phase Equilib. 22, 26 (2001).

[57] V. Vonk, D. Pontoni, M. Cremers, A. Kerkenaar, A. A. C. Bode, W. Szweryn, G. Nowak, A. E. F. de Jong, H. Dosch, and E. Vlieg, Langmuir 33, 814 (2017).

[58] J. Sudijono, M. D. Johnson, M. B. Elowitz, C. W. Snyder, and B. G. Orr, Surf. Sci 280, 247 (1993). 